\title{
Forces encountered by a sphere during impact into sand
}

\author{
Sylvain Joubaud, ${ }^{1, *}$ Tess Homan, ${ }^{2}$ Y. Gasteuil, ${ }^{3}$ Detlef Lohse, ${ }^{2}$ and Devaraj van der Meer ${ }^{2}$ \\ ${ }^{1}$ Laboratoire de Physique de l'École Normale Supérieure de Lyon, CNRS, Université de Lyon, F-69364 Lyon, France \\ ${ }^{2}$ Physics of Fluids Group, University of Twente, P.O. Box 217, 7500 AE Enschede, Netherlands \\ ${ }^{3}$ smartINST S.A.S., 213 rue de Gerland, 69007 Lyon, France
}

(Received 27 January 2014; revised manuscript received 18 June 2014; published 15 December 2014)

\begin{abstract}
We describe direct measurements of the acceleration of an object impacting on a loosely packed granular bed under various pressures, using an instrumented sphere. The sphere acts as a noninvasive probe that measures and continuously transmits the acceleration as it penetrates into the sand, using a radio signal. The time-resolved acceleration of the sphere reveals the detailed dynamics during the impact that cannot be resolved from the position information alone. Because of the unobstructed penetration, we see a downward acceleration of the sphere at the moment the air cavity collapses. The compressibility of the sand bed is observed through the oscillatory behavior of the acceleration curve for various ambient pressures; it shows the influence of interstitial air on the compaction of the sand as a function of time.
\end{abstract}

DOI: 10.1103/PhysRevE.90.060201

PACS number(s): 45.70.-n, 06.30.Gv, 45.40.-f, 47.57.Gc

Recently there has been growing interest in measuring the movement of objects in complex fluids such as granular systems [1,2], cornstarch suspensions [3], or foams [4]. The ultimate goal is to measure and understand the forces that act on an intruder in such fluids. Even if the global behavior is described by the bulk properties, it still lacks the details originating from the discrete nature of the material. Nonintrusive direct force measurements are difficult and in opaque systems forces cannot be derived from the trajectory of the object.

In this paper we study impact on dry quicksand, which is a specific granular state characterized by the fact that it cannot support any weight [5-7]. Previously, the trajectory of a ball inside such a sand bed was studied using a thin thread with markers attached to the ball [8-10] or using twodimensional experiments [11-13]. However, these methods are not accurate enough to determine the forces that act on the ball (i.e., proportional to the second time derivative of the trajectory), because the details of the acceleration data are smoothed out. Direct measurements of the sphere's acceleration in a more compacted bed have been performed using an accelerometer [14-16]; however, in those experiments the signal was transmitted via a plug attached to the sphere, which disturbs the sphere's motion after cavity closure.

Here we perform direct and noninvasive measurements of the acceleration. The idea is to send a sphere in an in-sand mission similar to the outer-space mission of a satellite: In both cases measurements are done and subsequently transmitted through radio signals. In both cases new terrain is explored and surprises are brought back from the mission.

The experimental setup [Fig. 1(a)] is similar to that of Refs. [8,9]: A container with a square cross section $(14 \times$ $14 \mathrm{~cm}^{2}$ ) and a height of $1.00 \mathrm{~m}$ is filled with nonspherical sand grains with sizes ranging from 20 to $60 \mu \mathrm{m}$. Subsequently, the sand bed is fluidized using pressurized dry air that is injected into the sand bed through a porous bottom plate. When slowly turning off the air flow, the bed settles into a very loose state with a packing fraction of approximately 0.41

*Corresponding author: sylvain.joubaud@ens-lyon.fr and a final bed height of $41 \mathrm{~cm}$ [8]. A sphere of diameter $D=2.5 \mathrm{~cm}$ is released with a pneumatic mechanism and dropped into the sand bed. At the moment of impact sand is ejected from the surface, which forms a crown-shaped splash. The ball then moves through the bed and a cavity, filled with air, is formed behind it. The walls of the cavity move inward and when they touch, two jets are formed, one upward (which eventually becomes visible above the bed) and a smaller one downward. The air below the pinch-off point is dragged along with the ball until it comes to a rest at a finite depth and the remaining air rises to the surface in an air bubble. There it causes an eruption, the final event visible above the surface. During the entire process the bed level lowers, meaning that the global packing fraction inside the bed is increased $[17,18]$. The initial drop height sets the impact velocity $v_{\text {impact }}$ and thus the Froude number $\mathrm{Fr}=2 v_{\text {impact }}^{2} / g D$, which is the ratio between characteristic kinetic and potential energies. Using a vacuum pump, the ambient air pressure $P_{0}$ within the container is varied between 10 mbars and 1 bar without affecting the packing structure of the sand bed [8].

The innovative component of the setup is the impacting object, an instrumented sphere that is designed and built by smartINST $^{\circledR}$ : The characteristics are described in detail in [19-21]. The basic properties of this system are depicted in Fig. 1(b). A three-axis acceleration sensor, an electrical circuit, and a button cell battery are all embedded in a spherical shell. The accelerometer measures the three components of the acceleration vector along the three perpendicular axes of the reference frame of the spherical particle. Each acceleration component can range from $-16 g$ to $16 g$, where $g$ is the gravitational acceleration $\left(g=9.8 \mathrm{~m} / \mathrm{s}^{2}\right)$. The resolution of acceleration is $0.01 \mathrm{~g}$ and the acquisition frequency is $500 \mathrm{~Hz}$ (i.e., a time resolution of $2 \mathrm{~ms}$ ). The signal is transmitted instantaneously using radio waves to a data acquisition device located next to the experiment.

A typical acceleration signal of the three accelerometers is presented in Fig. 2(a) for a Froude number of $\mathrm{Fr}=83$ and at atmospheric pressure $P_{0}=1$ bar. To be able to interpret these data in terms of the force of the sand acting on the particle, some approximations have to be made. The acceleration signal $\mathbf{a}_{M}$, measured in the frame of reference of the particle, is related 


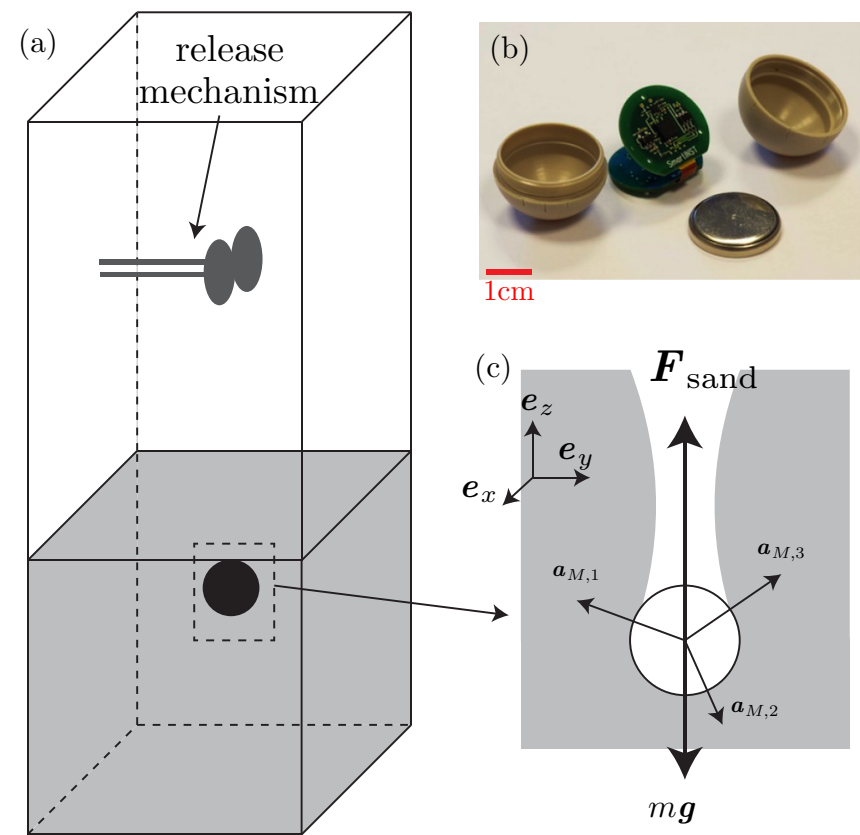

FIG. 1. (Color online) (a) Schematic of the experimental setup consisting of a container filled with fine, loose sand and a pneumatic release mechanism to controllably drop the instrumented particle from various heights. (b) Picture of the instrumented sphere (taken from [20]) with a diameter of $D=2.5 \mathrm{~cm}$. The particle contains three accelerometers and the required electronics to transfer the measurement signal to the outside world. (c) The three components of the measured acceleration signal in the frame of reference of the particle.

to the acceleration in the laboratory frame by

$$
\mathbf{a}_{M}=\mathbf{a}-\mathbf{g}+\mathbf{a}_{\epsilon} .
$$

The gravity term comes from the design of the accelerometer, which is such that the acceleration of the sphere at rest is equal to $\mathbf{- g}$ and equal to zero during free fall. The supplementary term $\mathbf{a}_{\epsilon}$ is mainly due to rotation $[20,21]$ and is so small that we can neglect it in the present study $\left(\left|\mathbf{a}_{\epsilon}\right| /|\mathbf{a}| \approx 1 \%\right)$. Assuming a unidirectional trajectory in the vertical direction $\mathbf{e}_{z}$, Eq. (1) may be written as a scalar equation and gives a direct relation between the magnitude of the measured acceleration and the force $\mathbf{F}_{\text {sand }}=F_{\text {sand }} \mathbf{e}_{z}$ of the sand on the particle (which is always in the upward, i.e., positive, direction)

$$
F_{\text {sand }}=m|\mathbf{a}-\mathbf{g}|=m\left|\mathbf{a}_{M}\right|=m a+m g,
$$

where $m$ is the mass of the particle, $\mathbf{a}=a \mathbf{e}_{z}$, and $\mathbf{g}=-g \mathbf{e}_{z}$. The velocity of the particle can now be obtained by integrating $a$ using the fact that the velocity needs to be zero when the particle is stopped (i.e., when $t>t_{\text {stop }}$ ): $v(t)=\int_{t}^{t_{\text {stop }}} a\left(t^{\prime}\right) d t^{\prime}$. Finally, the integration of the velocity signal gives the position $z$ of the particle as a function of time $z(t)=\int_{0}^{t} v\left(t^{\prime}\right) d t^{\prime}$. Here $t=0$ is defined at the time of impact, which corresponds to $z=0$.

This procedure was followed with the data of Fig. 2(a), with the measured acceleration signal $\left|\mathbf{a}_{M}(t)\right|=F_{\text {sand }} / m$ plotted in Fig. 2(b), the velocity $v(t)$ in Fig. 2(c), and the position $z(t)$ in Fig. 2(d). We will now discuss the different parts of acceleration signal $\left|\mathbf{a}_{M}(t)\right|$. Shortly after release of the
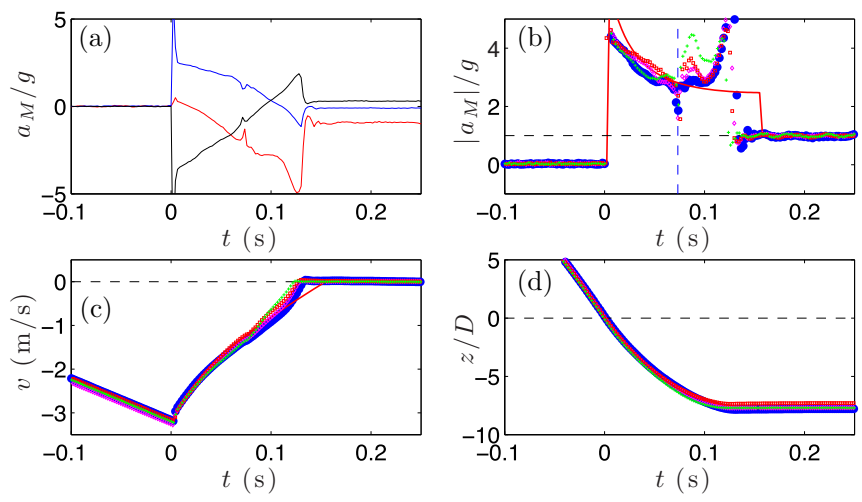

FIG. 2. (Color online) (a) Typical acceleration signals $a_{M, 1}, a_{M, 2}$, and $a_{M, 3}$ of the three sensors for an impact with $\mathrm{Fr}=83$ and $P_{0}=1$ bar. Accelerations are nondimensionalized with $g$. (b) Dimensionless measured acceleration $\left|\mathbf{a}_{M}\right| / g$ as a function of time. The small magenta, green, and red symbols represent an experiment done for the same external conditions to give an indication of the reproducibility of the experiment, which is good in the initial and medium phases. The vertical dashed blue line indicates the position of the peak, which is a signature of the jet formation. The solid red line is the fit of the position obtained using the model of the drag law (3) with $\kappa_{D}=3.5 \mathrm{~N} / \mathrm{m}$ and $\alpha=0.16 \mathrm{~kg} / \mathrm{m}$. (c) Particle velocity $v(t)=\int_{t}^{t_{\text {top }}} a\left(t^{\prime}\right) d t^{\prime}$ as a function of time $t$. The lines are the same as in (b). (d) Particle position $z(t)=\int_{0}^{t} v\left(t^{\prime}\right) d t^{\prime}$ (nondimensionalized with the particle diameter $D$ ) as a function of $t$. Note that the detailed information in $a(t)$ is smoothed out and thus lost in $z(t)$. The lines are the same as in (b).

sphere the nondimensionalized acceleration signal $\left|\mathbf{a}_{M}\right| / g$ goes to 0 , indicating free fall. When the sphere hits the sand the acceleration signal changes abruptly. Subsequently, the acceleration evolves during the motion of the sphere inside the sand until $\left|\mathbf{a}_{M}\right| / g$ suddenly drops to 1, indicating that the sphere has come to a stop and is experiencing a static friction force that is equal in magnitude but opposite to gravity. Note that (due to the double integration) the position signal bears no sign of the short-time variations that are so visible in the acceleration signal. In general, features that appear to be significant in the acceleration signal may be hard to discern (if at all) in the time evolution of velocity and position.

As one can argue that these variations may be due to errors in the measurement signals, measurements done under the same conditions (Froude number and ambient pressure) have been repeated several times. To clearly distinguish which features are robust and which are not, in all the following figures, a second experiment is plotted using the small magenta symbols. In Fig. 2 the reproducibility is very good until the peak at around $80 \mathrm{~ms}$. After that, the behavior is substantially different for each experiment: There is variation in the deceleration magnitude and even in the stopping time.

First, we compare these experimental results with the phenomenological drag law introduced by Katsuragi [22,23] to fit the position as a function of time [8,9]. This law consists of a hydrostatic term that linearly depends on the depth inside the bed $-\kappa_{D} z$, where $\kappa_{D}$ depends only on the ambient air pressure [8], and a second term that is a quadratic velocity-dependent drag force that is independent of the depth $-\alpha v^{2}$ [24],

$$
F_{\text {sand }}=-\kappa_{D} z-\alpha v^{2}
$$



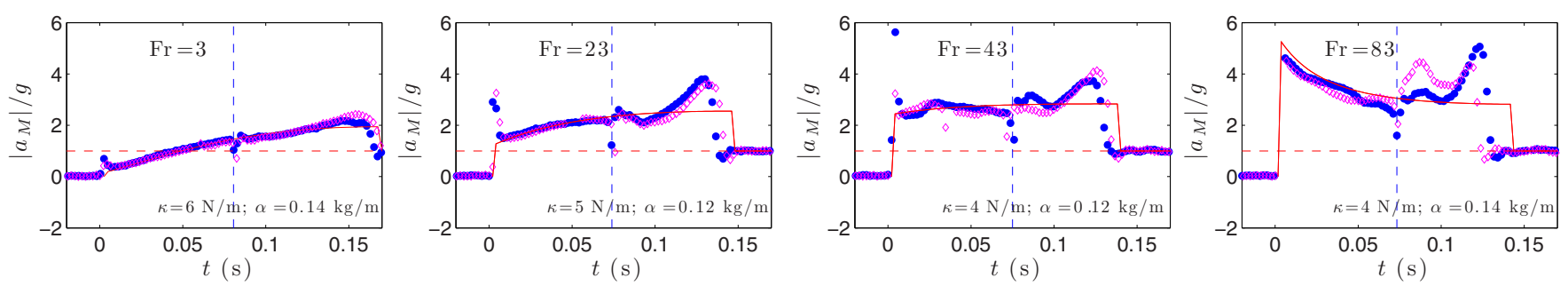

FIG. 3. (Color online) Measured accelerations for different impact velocity (or Froude number) at ambient pressure $\left(P_{0}=1\right.$ bar): (a) $\mathrm{Fr}=3$, (b) $\mathrm{Fr}=23$, (c) $\mathrm{Fr}=43$, and (d) $\mathrm{Fr}=83$. The red solid line is the phenomenological model (3) obtained from the fit of the trajectory. The blue and magenta symbols represent two experiments done for the same external conditions. The vertical dashed blue lines indicate the position of the peak connected to the jet formation.

In Fig. 2(d) the particle position data are very well fitted by the solution of $m \ddot{z}=-m g+F_{\text {sand }}$ using the full drag force law (3) indicated by the solid red line. However, when we turn to the acceleration data [Fig. 2(b)], we see that the fit is much poorer. Before the peak at $80 \mathrm{~ms}$ there is a reasonable agreement between the phenomenological model and the experiment, but for later times there are large discrepancies. The model correctly describes the global behavior, but the short-time-scale irregularities that appear in the acceleration signal are clearly beyond the capacity of such a model. In the following figures, the fit with the model of Eq. (3) is always plotted together with the acceleration signal.

We will now focus on those features that can only be seen in the acceleration data and are missed in the simplistic model. In Fig. 3 experiments at different Froude numbers (i.e., different impact speeds) are presented and it is clear that especially the short-time-scale features, i.e., directly after impact, strongly depend on Fr. The higher Fr is, the stronger the initial deceleration peak that disappears when the impact velocity is close to zero. This first phenomenon is consistent with previous experiments done by [15]. Inside the bed the mean deceleration increases with increasing Froude number, as predicted by the phenomenological drag law.

Since the effect of air inside the bed is found to be a key point in the sand bed dynamics [8,9], experiments are repeated for various ambient air pressures and are shown in Fig. 4 for $\mathrm{Fr}=3$ and 83 . The first thing to note is that the mean value of the deceleration increases for decreasing ambient pressure. This is consistent with the increase of the drag force constant $\kappa$ measured in [8], which also showed an increasing drag on the ball for lower ambient pressures. The variations of the measurements around the deceleration expected from the model (the differences between the blue experimental points and the red solid line in Fig. 4) become more pronounced for lower ambient pressures. In the model
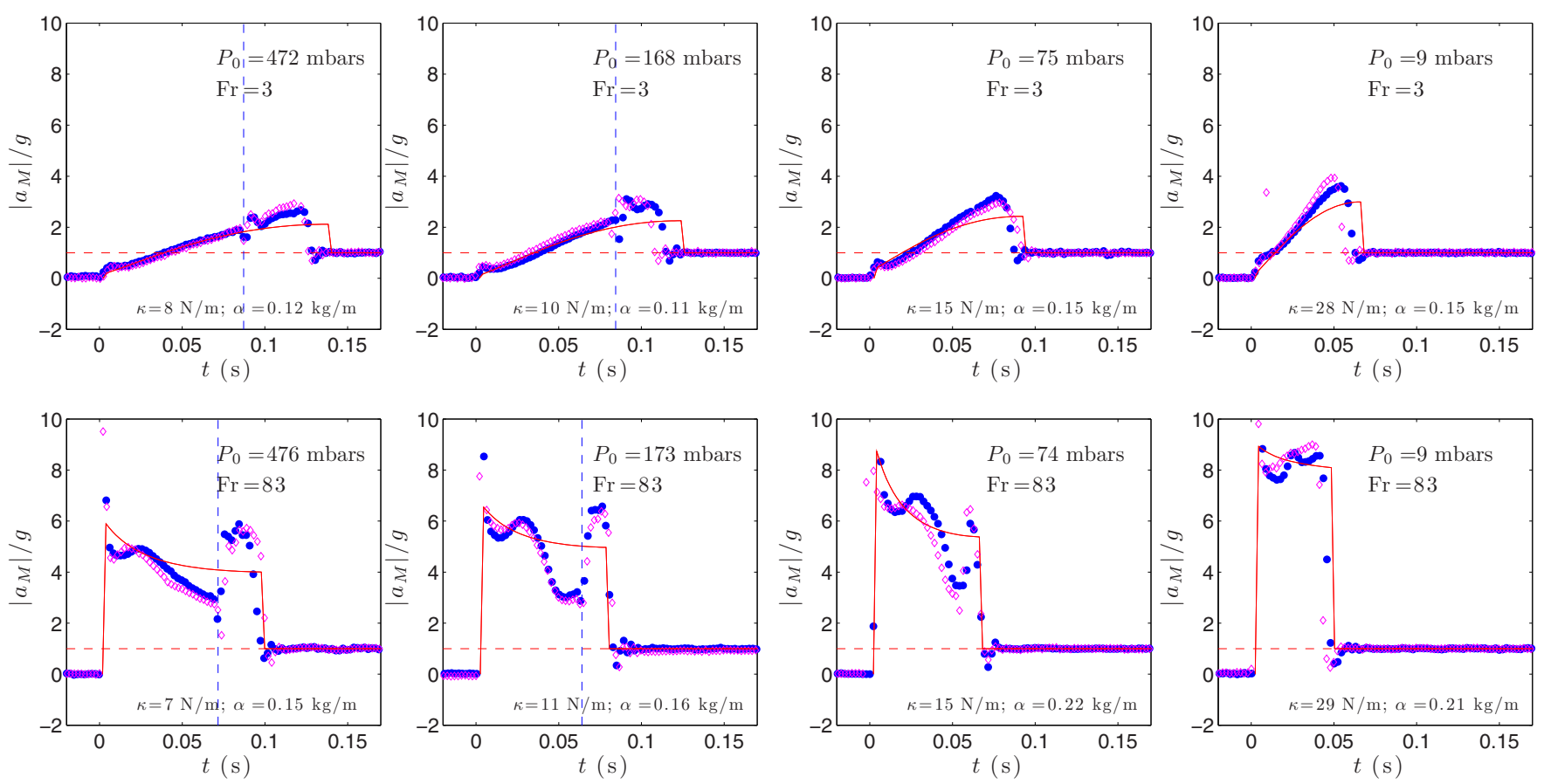

FIG. 4. (Color online) Measured accelerations for two Froude numbers $\mathrm{Fr}=3$ and 83 and different ambient pressure: (a) $P_{0}=476 \mathrm{mbars}$, (b) $P_{0}=173$ mbars, (c) $P_{0}=74$ mbars, and (d) $P_{0}=9$ mbars. The red solid lines again result from the phenomenological model (3) obtained from the fit of the trajectory. The blue and magenta symbols represent two experiments. The vertical dashed blue lines indicate the position of the peak connected to the jet formation. 
the sand bed is expected to behave as an incompressible fluid, but these growing variations show that this assumption becomes less valid with reduced pressure. At lower ambient pressures the sand around the ball is compacted more [18], therefore the magnitude of the variations is a measure for the compressibility of the system. Thus, by probing a granular system with an impactor, it is possible to extract information about the influence of air on the compressibility of the bed, locally and for the bed as a whole. When there is less air present to counteract the compaction caused by the falling ball, the drag on the ball not only increases, but, surprisingly, also departs more significantly from the drag law (3). This means that there is a compacting-decompacting mechanism present that has not yet been seen in dry granular materials.

At around $80 \mathrm{~ms}$ after impact the sphere is briefly accelerated instead of slowed down for almost all experimental conditions (see Figs. 3 and 4). This acceleration peak appears at a time that coincides with the collapse of the cavity and therefore is a signature of the jet formation. Since the duration of the cavity collapse is expected to be very short (shorter than our time resolution of $2 \mathrm{~ms}$ ), we only see an averaged signal of the collapse and it is not possible to fully resolve it. However, it is evident that the sphere at this moment is accelerated and we suggest two possible mechanisms. First, the downward granular jet can actually impact the sphere and push it towards the bottom of the container (thereby increasing $\left.F_{\text {sand }}\right)$. Second, the air bubble that is entrapped when the cavity collapses is pressurized, which may also induce a downward force. Only for very low ambient pressures (below 100 mbars) the peak vanishes in our measurements. For those cases the cavity typically closes after the sphere has stopped [8] and this process will no longer be able to accelerate the sphere. The cavity collapse is directly observed through measuring the acceleration of the impact object, confirming the valuable properties of the smart particle device [26].

After the peak, the measured deceleration is higher than before the peak. Two mechanisms contribute to the larger drag that is experienced by the sphere after the collapse of the cavity. The first one is the increase of the packing fraction in front of the ball and in the entire bed just after the collapse as mentioned in Refs. [17,27]. At atmospheric pressure it is speculated by $[8,18,28]$ that the compaction is (partially) prevented by an increase of the interstitial air pressure or by a local fluidization mechanism. At lower pressures the compaction is more pronounced, leading to a larger drag and a larger deceleration, as can be seen in Fig. 4. The second possible origin lies in the air bubble that is pinched off at the collapse and is attached to the sphere during its motion inside the sand. The absence of grains on top of the sphere effectively reduces the downward force component of the (upward) drag, resulting in a larger deceleration. Since the dynamics and shape of the pinched-off air bubble is clearly nonreproducible [17], the dragging along of the air bubble may also be an explanation for the differences between repeated measurements for the same conditions. This explanation is supported by the observation that, as can be seen in Figs. 3 and 4 , the results at low Froude number and low pressure (where air plays less of a role) are more reproducible.

Finally, we would like to point out a small overshoot that is visible in the signal when the ball comes to a rest, similar to the one seen in [16]. There it was interpreted as a signature of the sand below the sphere behaving like an elastic solid. The present resolution is however insufficient to confirm this interpretation.

In summary, we have provided direct measurements of the acceleration of a sphere impacting on a loosely packed granular bed, using a noninvasive measurement technique. The model of Katsuragi [23] correctly predicts the shape of the position data as a function of time, but it cannot describe the short time-scale features in the acceleration signals. Signatures of the cavity collapse and the compressibility of the sand bed for small ambient pressures were measured. The existence of these features and how they change with varying Froude number and ambient pressures help us to understand what happens inside the sand bed. However, a thorough theoretical interpretation that incorporates knowledge of the influence of the interstitial air and the interactions at the grain scale is still needed.

The smart particle device used in these measurements proved to be an invaluable tool. These real-time acceleration measurements may be used in many other applications where this type of Lagrangian measurement is otherwise hardly feasible. It is especially useful in opaque media, which can be found in, e.g., avalanches, foam studies, or the pharmaceutical industry. The present paper provides additional evidence that noninvasive, direct acceleration measurements give better insight into the complicated underlying dynamics than the trajectories alone.

This work is part of the research program of the Foundation for Fundamental Research on Matter (FOM), which is part of the Netherlands Organisation for Scientific Research (NWO).
[1] F. Guillard, Y. Forterre, and O. Pouliquen, Phys. Rev. Lett. 110, 138303 (2013).

[2] J. C. Ruiz-Suarez, Rep. Prog. Phys. 76, 066601 (2013).

[3] S. von Kann, J. H. Snoeijer, and D. van der Meer, Phys. Rev. E 87, 042301 (2013).

[4] B. Dollet, F. Elias, C. Quilliet, C. Raufaste, M. Aubouy, and F. Graner, Phys. Rev. E 71, 031403 (2005).

[5] D. Lohse, D. Rauhé, R. P. H. M. Bergmann, and D. van der Meer, Nature (London) 432, 689 (2004).

[6] S. T. Thoroddsen and A. Q. Shen, Phys. Fluids 13, 4 (2001).
[7] J. R. Royer, E. I. Corwin, A. Flior, M.-L. Cordero, M. L. Rivers, P. J. Eng, and H. M. Jaeger, Nat. Phys. 1, 164 (2005).

[8] G. Caballero, R. Bergmann, D. van der Meer, A. Prosperetti, and D. Lohse, Phys. Rev. Lett. 99, 018001 (2007).

[9] S. von Kann, S. Joubaud, G. A. Caballero-Robledo, D. Lohse, and D. van der Meer, Phys. Rev. E 81, 041306 (2010).

[10] M. Hou, Z. Peng, R. Liu, K. Lu, and C. K. Chan, Phys. Rev. E 72, 062301 (2005).

[11] W. A. Allen, E. B. Mayfield, and H. L. Morrison, J. Appl. Phys. 28, 1331 (1957). 
[12] M. P. Ciamarra, A. H. Lara, A. T. Lee, D. I. Goldman, I. Vishik, and H. L. Swinney, Phys. Rev. Lett. 92, 194301 (2004).

[13] F. Pacheco-Vazquez, G. A. Caballero-Robledo, J. M. SolanoAltamirano, E. Altshuler, A. J. Batista-Leyva, and J. C. RuizSuarez, Phys. Rev. Lett. 106, 218001 (2011).

[14] Y. Boguslavskii, S. Drabkin, and A. Salman, J. Phys. D 29, 905 (1996).

[15] P. Umbanhowar and D. I. Goldman, Phys. Rev. E 82, 010301 (2010).

[16] D. I. Goldman and P. Umbanhowar, Phys. Rev. E 77, 021308 (2008).

[17] T. Homan, R. Mudde, D. Lohse, and D. van der Meer, arXiv:1412.2907 [J. Fluid Mech. (to be published)].

[18] J. R. Royer, B. Conyers, E. I. Corwin, P. J. Eng, and H. M. Jaeger, Europhys. Lett. 93, 28008 (2011).

[19] S. Harada, Y. Kobayashi, T. Sawano, and E. Noguchi, Int. J. Multiphase Flow 37, 898 (2011).

[20] R. Zimmermann, L. Fiabane, Y. Gasteuil, R. Volk, and J.-F. Pinton, New J. Phys. 15, 015018 (2013).

[21] R. Zimmermann, L. Fiabane, Y. Gasteuil, R. Volk, and J.-F. Pinton, Phys. Scr. T155, 014063 (2013).
[22] L. S. Tsimring and D. Volfson, in Powders and Grains 2005, edited by R. Garcia-Rojo, H. J. Herrmann, and S. McNamara (Taylor \& Francis, London, 2005), p. 1215-1223

[23] H. Katsuragi and D. J. Durian, Nat. Phys. 3, 420 (2007).

[24] Using direct acceleration measurements, Goldman and Umbanhowar $[15,16]$ developed a drag law using a term linear with velocity as an alternative to the second term in the Katsuragi law. Such a linear term had also been proposed earlier in [25].

[25] J. R. de Bruyn and A. Walsh, Can. J. Phys. 82, 439 (2004).

[26] The effect of the granular jet could not be seen in previous experiments by measuring the position or by using the device of [15] in which the accelerometer was mounted on an aluminum plug. Such a device would be of no use in our experiment because the jet appears before the stopping time and would be destroyed by the presence of the plug.

[27] T. Homan, C. Gjaltema, and D. van der Meer, Phys. Rev. E 89, 052204 (2014).

[28] J. R. Royer, E. I. Corwin, B. Conyers, A. Flior, M. L. Rivers, P. J. Eng, and H. M. Jaeger, Phys. Rev. E 78, 011305 (2008). 